\begin{tabular}{|c|c|}
\hline Title & $\begin{array}{l}\text { Strong and Tough Polyion-Complex Hydrogels from Oppositely Charged Polyelectrolytes: A Comparative Study with } \\
\text { Polyampholyte Hydrogels }\end{array}$ \\
\hline Author(s) & $\begin{array}{l}\text { Luo, Feng; Sun, Tao Lin; Nakajima, Tasuku; King, Daniel R.; Kurokawa, Takayuki; Zhao, Y Y; Ihsan, A bu Bin; Li, } \\
\text { X ufeng; Guo, Honglei; Gong, Jian Ping }\end{array}$ \\
\hline Citation & $\begin{array}{l}\text { Macromolecules, 49(7), 2750-2760 } \\
\text { https://doi.org/10.1021/acs.macromol.6b00235 }\end{array}$ \\
\hline Issue Date & $2016-04-12$ \\
\hline Doc URL & http:/hdl .handle.net/2115/64952 \\
\hline Rights & $\begin{array}{l}\text { This document is the A ccepted Manuscript version of a Published Work that appeared in final form in Macromolecules, } \\
\text { copyright } \odot 2016 \text { A merican Chemical Society after peer review and technical editing by the publisher. To access the } \\
\text { final edited and published work see http://pubs.acs.org/doi/abs/10.1021/acs.macromol.6b00235 }\end{array}$ \\
\hline Type & article (author version) \\
\hline File Information & Strong and Tough.pdf \\
\hline
\end{tabular}

Instructions for use 
This document is confidential and is proprietary to the American Chemical Society and its authors. Do not copy or disclose without written permission. If you have received this item in error, notify the sender and delete all copies.

\section{Strong and Tough Polyion-Complex Hydrogels from Oppositely Charged Polyelectrolytes: A Comparative Study with Polyampholyte Hydrogels}

\begin{tabular}{|r|l|}
\hline Journal: & Macromolecules \\
\hline Manuscript ID & ma-2016-00235y.R1 \\
\hline Manuscript Type: & Article \\
\hline Domplete List of Authors: & $\begin{array}{l}\text { Luo, Feng; Faculty of Advanced Life Science, } \\
\text { Sun, Tao Lin; Hokkaido University, Graduate School of Science } \\
\text { Nakajima, Tasuku; Hokkaido University, Faculty of Advanced Life Science } \\
\text { King, Daniel; Hokkaido University, Faculty of Advanced Life Science } \\
\text { Kurokawa, Takayuki; Faculty of Advanced Life Science, Hokkaido University } \\
\text { Zhao, Yu; Graduate School of Life Science, Hokkaido University } \\
\text { Ihsan, Abu Bin; Hokkaido University, Graduate School of Science } \\
\text { Li, Xufeng; Hokkaido University, } \\
\text { Guo, Honglei ; Graduate School of Life Science, Hokkaido University } \\
\text { Gong, Jian Ping; Hokkaido University, Graduate School of Science }\end{array}$ \\
\hline
\end{tabular}




\title{
Strong and Tough Polyion-Complex Hydrogels from Oppositely Charged Polyelectrolytes: A Comparative Study with Polyampholyte Hydrogels
}

Feng Luo ${ }^{1, \$}$, Tao Lin Sun ${ }^{1,2}$, Tasuku Nakajima ${ }^{1,2}$, Daniel R. King ${ }^{1,2}$, Takayuki Kurokawa $^{1,2}$, Yu Zhao ${ }^{3}$, Abu Bin Ihsan ${ }^{1}$, Xufeng Li $^{3}$, Honglei Guo ${ }^{3}$, Jian Ping Gong ${ }^{1,2^{*}}$

${ }^{1}$ Faculty of Advanced Life Science, Hokkaido University, Sapporo 060-0810, Japan

${ }^{2}$ Global Station for Soft Matter, Global Institution for Collaborative Research and Education, Hokkaido University, Sapporo, Japan

${ }^{3}$ Graduate School of Life Science, Hokkaido University, Sapporo 060-0810, Japan

*Corresponding author: gong@mail.sci.hokudai.ac.jp

\$: Present Address

F. Luo: College of Polymer Science and Engineering, State Key Laboratory of Polymer Materials Engineering, Sichuan University, Chengdu 610065, China

\begin{abstract}
Oppositely charged homo-polyelectrolytes were found to form strong, tough, and selfhealing polyion-complex (PIC) hydrogels, in similar to polyampholytes (PA) that have opposite charges randomly distributed on the same polymer chains. The excellent mechanical performances of these two novel hydrogels are the results of dynamic ionic bonds formation between entangled polymer chains. For PIC system, only inter-chain bonding occurs, while for PA system both inter- and intra-chain bonding exist. In addition, the ion pairs are expected to form stronger bonding in PIC system than those in PA system. In this work, we performed a comparative study of PIC hydrogels with the PA hydrogels. The PIC hydrogels are synthesized by sequential homo-polymerization of
\end{abstract}


cationic and anionic monomers at varied formulation, and their swelling and mechanical properties are systematically studied in comparison to the PA hydrogels that were synthesized from random copolymerization of anionic monomers and cationic monomers of the similar formulation. Different from the PA system that only forms tough hydrogels around zero-net charge composition without chemical cross-linking, the PIC system forms tough physical hydrogels even at weakly off-balanced charge composition. At the charge-balanced composition, the low entanglement concentration of homo-charged polyelectrolyte chains leads to tough PIC hydrogels formation at much lower concentrations than that of PA hydrogels. As a result, the PIC hydrogels are much tougher than the PA hydrogels prepared at the same monomer composition. In similar to PA hydrogels, the PIC hydrogels also exhibit broad dynamic mechanical spectra, indicating the formation of ion complexes with widely ranged bond strength. The PIC hydrogels have strong viscoelasticity in comparison with PA hydrogels. However, the two systems show the similar activation energies of the dynamic mechanical spectra. The SEM microstructural observation shows that the PIC hydrogels have segregated structure while PA hydrogels are more homogeneous. 


\title{
for Table of Contents use only
}

\section{Strong and Tough Polyion-Complex Hydrogels from Oppositely Charged}

Polyelectrolytes: A Comparative Study with Polyampholyte Hydrogels

\author{
Feng Luo ${ }^{1, \$}$, Tao Lin Sun ${ }^{1,2}$, Tasuku Nakajima ${ }^{1,2}$, Daniel R. King ${ }^{1,2}$, Takayuki
}

Kurokawa ${ }^{1,2}$, Yu Zhao ${ }^{3}$, Abu Bin Ihsan ${ }^{1}$, Xufeng $\mathrm{Li}^{3}$, Honglei Guo ${ }^{3}$ and Jian Ping

Gong $^{1,2^{*}}$

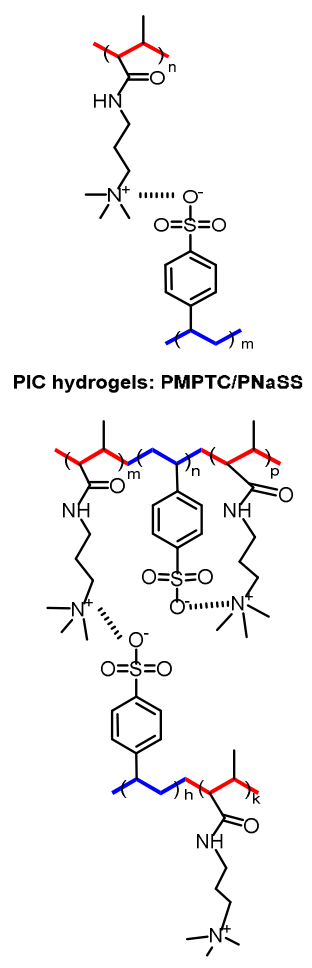

PA hydrogels: P(MPTC-co-NaSS)
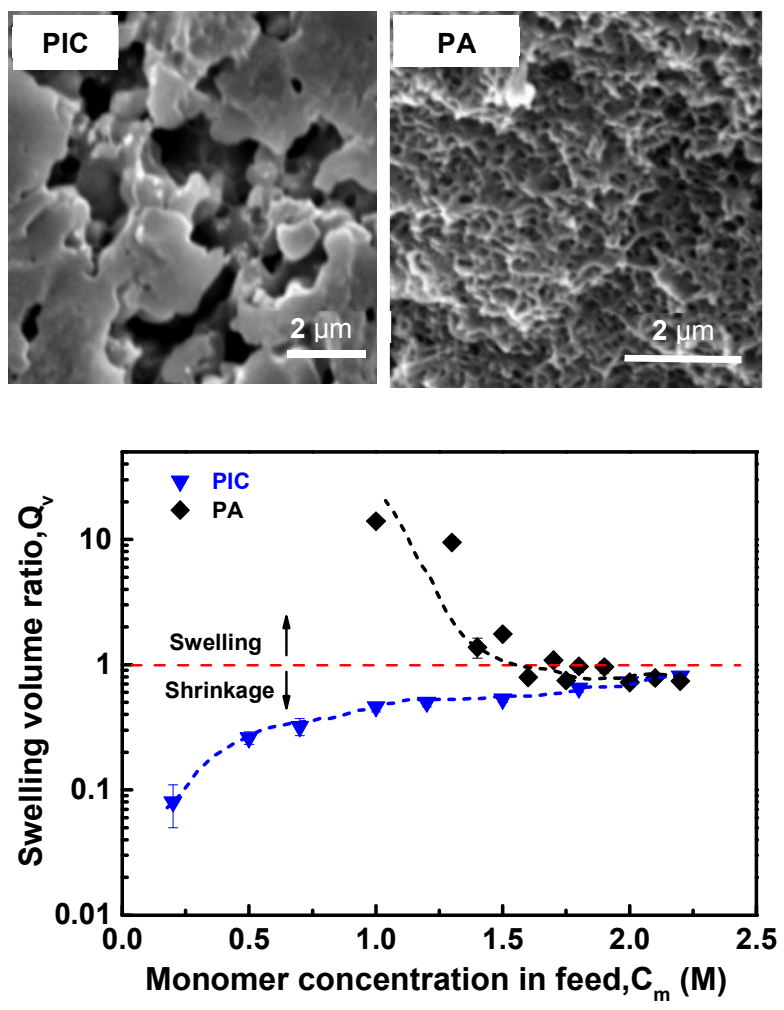


\section{Introduction}

Hydrogels are "soft and wet" materials that possess unique properties necessary for application in biomaterials, soft robotics, smart actuators, and sensors. ${ }^{1-8}$ The applicability of hydrogels, however, is often severely limited by their weak and brittle mechanical properties, which are due to intrinsic structural inhomogeneity and lack of an effective energy dissipation mechanism. Therefore, developing robust hydrogels with excellent mechanical properties and functionality has long been pursued by material scientists. In nature, tough and strong hydrogels, like natural, load-bearing soft tissues such as tendon, cartilage, muscle, and blood vessels, have been used to inspire soft materials design. During the past few decades, much effort has been devoted to developing tough hydrogels, such as double-network hydrogels, ${ }^{9}$ nanocomposite hydrogels, ${ }^{10-11}$ hybrid hydrogels ${ }^{8,12-13}$, and others. ${ }^{14-15}$ Among them, the double network (DN) strategy has been shown to be extraordinarily effective in improving the toughness of hydrogels, and the "sacrificial bond" mechanism was shown to efficiently dissipate energy ${ }^{16}$ Since the rupture of covalent bonds in traditional DN gels is irreversible, DN gels exhibit permanent softening after large deformation, which is a limitation in many practical applications. To address this problem, physically reversible bonds can be built in the hydrogel as a renewable "sacrificial bond" to replace the irreversible covalent bonds. Hydrogen bonds, ${ }^{17}$ metal-ligand interactions, ${ }^{18}$ ionic bonds, ${ }^{19-20}$ charge-driven self-assembling, ${ }^{21-23}$ Van der Waals interactions, ${ }^{24} \pi-\pi$ interactions, ${ }^{25}$ and hydrophobic interactions, ${ }^{26}$ are all potential candidates as a renewable "sacrificial bond." Studies along these lines have successfully produced tough double network hydrogels with selfrecovery after internal rupture. ${ }^{1,19,27}$ 
The double network concept, incorporating a mechanically fragile structure to toughen the entire material, suggests a more general strategy of incorporating dynamic physical bonds for developing tough hydrogels. Recently, our group developed two kinds of robust and self-healing physical hydrogels using dynamic ionic bonds. One is polyampholyte (PA) hydrogels from one-step free radical copolymerization of chargebalanced anionic and cationic monomers. ${ }^{28-30}$ Another is polyion-complex (PIC) hydrogels from sequential homopolymerization of opposite charged cationic and anionic monomers. ${ }^{31-32}$ In these gels, dynamic ionic bonds, of wide distribution in strength, are formed. The strong bonds serve as crosslinkers, imparting elasticity, whereas the weak bonds serve as reversible sacrificial bonds, imparting toughness and self-healing.

Considering that both PIC hydrogels and PA hydrogels are formed from oppositely charged monomers but with different primary structures of polymers through different synthesizing processes, it is interesting to compare the behaviors of these two systems. For example, both inter- and intra-chain bonding exist in the PA system, while PIC system only has inter-chain bonding. How does this difference influence the stability of the ionic bond strength? In the case of PA hydrogels, the primary structure of the polymer strongly depends on the reactivity ratios of the two oppositely charged monomers, which makes it a complex system to reveal the detailed correlation between the structure and the properties of these gels. In contrast, PIC hydrogels, consisting of oppositely charged homo-polymers, are relatively simple to study. By comparing the behaviors of the two systems, we might obtain insight to understand the PA system.

Previous studies have shown that the charge ratio and the monomer concentration are two important factors influencing PA hydrogel structure and properties. ${ }^{28,30}$ In this 
work, we systematically study the effects of charge ratio, the monomer concentrations, and the molecular weight of polyelectrolytes on the swelling, dynamic mechanical response, and mechanical performance of PIC hydrogels via a comparative study with PA hydrogels synthesized from the same pair of cationic and anionic monomers.

\section{Experimental section}

\subsection{Materials}

Commercially available anionic monomer, sodium $p$-styrenesulfonate (NaSS); cationic monomer, 3-(methacryloylamino)propyl-trimethylammonium chloride (MPTC); UV initiator, $\alpha$-ketoglutaric acid; and $\mathrm{NaCl}$ are all purchased from Wako Pure Chemical Industries, Ltd. and used as received. Millipore de-ionized water is used in all of the experiments.

\subsection{Synthesis of hydrogels}

Polyion complex hydrogels. The PIC hydrogel was synthesized by sequential homopolymerization of cationic and anionic monomers, as shown in Scheme 1a, which is the same method as that employed in the previous report. ${ }^{31}$ Typically, the sample is referred to as $\mathrm{PM}_{1} / \mathrm{PM}_{2}\left(C_{m}-f-\mathrm{x}_{1}-\mathrm{x}_{2}\right)$, where $\mathrm{P}$ is the abbreviation of poly-, and $\mathrm{M}_{\mathrm{i}},(\mathrm{i}=1,2)$ is the abbreviated monomer name in the ith step of the polymerization. $C_{m}$ is the overall molar concentration at the second component polymerization, $f$ is the molar fraction of anionic monomer, and $\mathrm{x}_{1}$ and $\mathrm{x}_{2}$ are initiator concentrations of the first and second polymerization $(\mathrm{mol} \%)$, relative to the corresponding monomers. For example, to synthesize the PMPTC/PNaSS(1.5-0.5-0.05-0.1) hydrogel, PMPTC was first synthesized from an aqueous solution of $1 \mathrm{M}$ MPTC containing $\mathrm{x}_{1}=0.05 \mathrm{~mol}-\%$ initiator, 2oxoglutaric acid, by UV light irradiation (light intensity $\sim 4 \mathrm{~mW} / \mathrm{cm}^{2}$ ) for $8 \mathrm{~h}$. The produced PMPTC was dried and made into a powder. Next the powder was mixed with NaSS monomer with the charge fraction of the second monomer NaSS, $f$ $=[\mathrm{NaSS}] /([\mathrm{MPTC}]+[\mathrm{NaSS}])=0.5$. After well dispersion with $0.5 \mathrm{M} \mathrm{NaCl}$ to form a 
mixed solution of $C_{m}=[\mathrm{MPTC}]+[\mathrm{NaSS}]=1.5 \mathrm{M}$ at $60{ }^{\circ} \mathrm{C}$, the mixture containing $\mathrm{x}_{2}=0.1$ mol-\% initiator relative to the second monomer was polymerized under UV light for $8 \mathrm{~h}$ in a reaction cell consisting of a pair of glass plates with $1.5 \mathrm{~mm}$ spacing. The asprepared hydrogel was then dialyzed in a large amount of water to remove the mobile counter ions and co-ions from the product. After dialysis, the opposite charges on the polymer chains formed ion bonds. Thus, the thickness of the sample shrank and the equilibrium hydrogel was obtained. Different $C_{m}$ from 0.2 to $2.2 \mathrm{M}$ and different molar fractions $f$ from 0.45 to 0.55 were investigated in this work.

Polyampholyte hydrogels. PA hydrogels P(MPTC-co-NaSS), synthesized from random copolymerization of anionic monomer NaSS and cationic monomer MPTC, as shown in Scheme $1 b$, was obtained according to our previous report. ${ }^{28,29,33}$ The mixed solution with prescribed total ionic monomer concentration $C_{m}$, molar fraction of anionic monomer $f, 0.25 \mathrm{~mol} \%$ initiator relative to $C_{m}$ was polymerized in a reaction cell with 2 mm spacing under UV light for $8 \mathrm{~h}$. To reach the equilibrated state, the as-prepared gels were also immersed in a large amount of water for one week, where the mobile counter ions and unreacted co-ions were dialysed from the sample. As the PA can only form physical hydrogels in a very narrow range of $f \sim 0.5$, the sample series with varied charge fraction $f$ were synthesized in the presence of chemical cross-linker, N, N'methylenebis(acrylamide) (MBAA) (4 mol\% in relative to monomer concentration $C_{m}$ ). The PA sample is referred to as P(MPTC-co-NaSS) $\left(C_{m}-f-C_{M B A A}\right)$, where $C_{m}$ is the overall molar concentration of monomer, $f$ is the molar fraction of anionic monomer, $C_{M B A A}$ is the chemical cross-linker density. For PA hydrogels without chemical cross-linker, the sample is referred to as $\mathrm{P}(\mathrm{MPTC}-\mathrm{co}-\mathrm{NaSS})\left(C_{m}-f\right)$.

The measured reactive ratio of NaSS and MPTC is 1.48 and 0.70 , respectively. ${ }^{28}$ So the sequence of the P(MPTC-co-NaSS) synthesized at balanced charge composition can be 
calculated as NaSS rich at the starting end of the chain and the MPTC rich at the finishing end of the chain. Also, some copolymers may not be neutral and those formed at the early stage is NaSS rich, while those at the later stage is MPTC rich. At the equilibrated state, the NaSS rich segments and MPTC rich segments would form the strong ionic complex structure as well as the entanglements, while other parts lead to the weak ionic complex. Therefore, it shows wide distribution of strong bonds and weak bonds. The strong bonds serve as permanent crosslinks, imparting the elasticity, while the weak bonds reversibly break and re-form, serving as reversible sacrificial bonds.

(a)

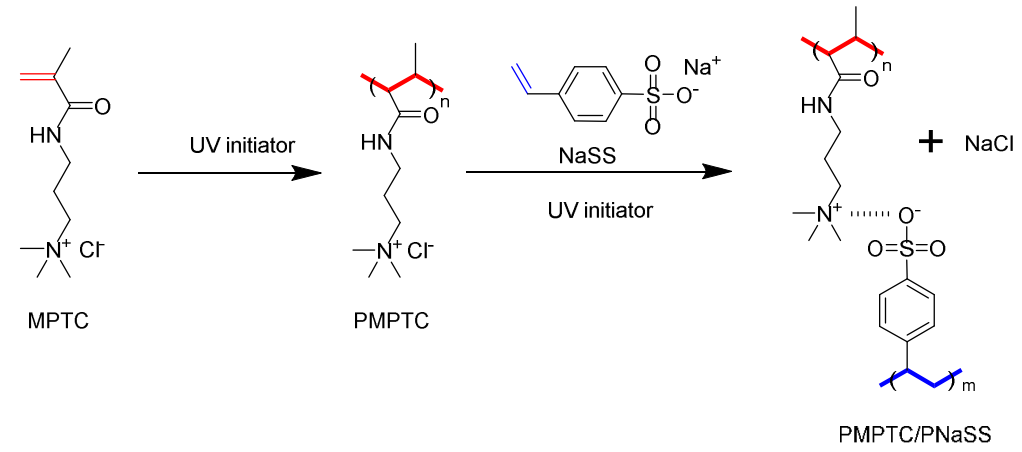

(b)

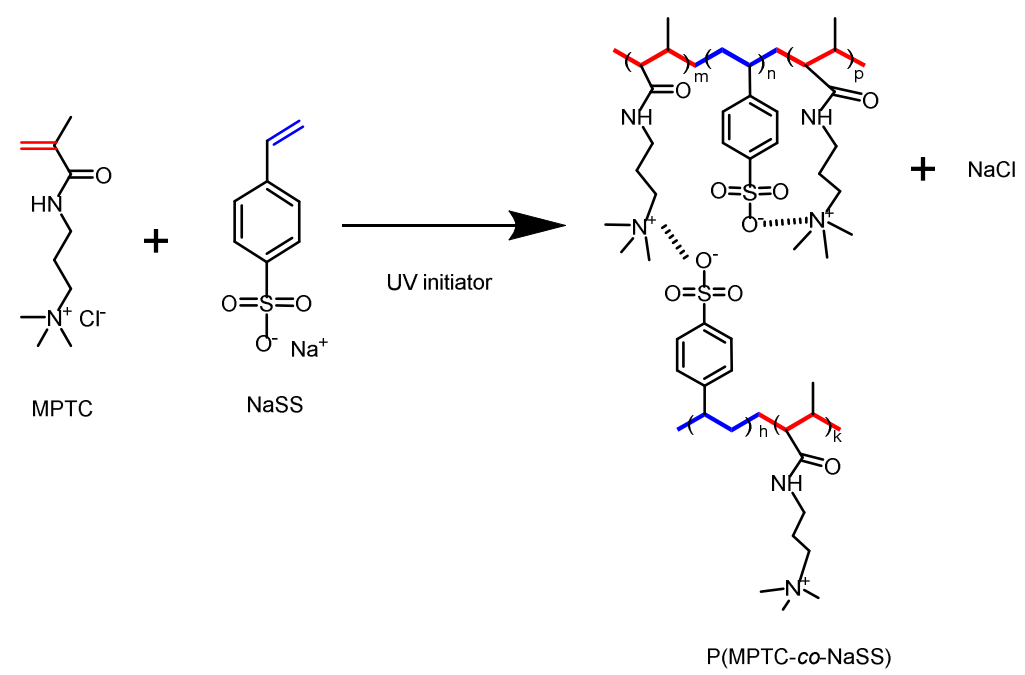

Scheme 1. Schematics of the preparation of the physical hydrogels by ionic bonds formation from oppositely charged homo-polyelectrolytes to form polyion-complex (PIC) 
or from polyampholytes (PA) that carrying opposite charges on the same polymer chain. (a) PIC hydrogel formation: Cationic monomer (MPTC) was homopolymerized in the first step and then mix with the anionic monomer (NaSS). After mixing, the latter monomer was polymerized in the second step to form the as-prepared PIC hydrogel. By equilibrating the sample in water, small counter-ions and co-ions of the polymer are removed from the sample. As a result, tough PIC hydrogel is formed, named PMPTC/PNaSS. (b) PA hydrogel P(MPTC-co-NaSS) formation: synthesized from onestep random copolymerization of cationic monomer MPTC and anionic monomer NaSS.

\subsection{Characterization}

Swelling measurements. The swelling volume ratio, $Q_{v}$, was defined as the ratio of the sample volume at swelling equilibrium, $V$, to that of the as-prepared state, $V_{0}, Q_{v}=V / V_{0}$. The polymer molar concentration, $C_{p}$, at equilibrium of the sample was estimated from $C_{m} / Q_{v}$. To achieve adequate precision, three measurements were carried out on samples of different volumes taken from the same gel. The water content of dialyzed samples was measured using a moisture tester.

Tensile test. The tensile tests were carried out on dumbbell-shaped samples with the standard JIS-K6251-7 size using a commercial tensile tester (Tensilon RTC-1310A, Orientec Co.). The initial distance $L_{0}$ between the two clamps of the tester was $12 \mathrm{~mm}$ and the tensile deformation was performed at a series of extension rates, $v$, from 1 to 100 $\mathrm{mm} / \mathrm{min}$., which gives the strain rate $\dot{\varepsilon}=v / L_{0}$ from 0.0014 to $0.14 \mathrm{~s}^{-1}$. The nominal stress, $\sigma$, was estimated from the load divided by the cross-sectional area of the undeformed sample. The strain $\varepsilon$ was estimated from the clamp displacement divided by $L_{0}$. The 
Young's modulus, E, was estimated as the slope of the stress-strain curve within a strain range of $\varepsilon=0.03-0.1$. The yield stress, $\sigma_{y}$, was obtained at which the tangent modulus $d \sigma / d \varepsilon$ changes at the greatest rate with respect to increasing strain. The values of all the mechanical properties were calculated as averages for at least three specimens. All of the measurements were performed in air at a room temperature of about $25^{\circ} \mathrm{C}$.

Rheometry. Rheological tests were performed using an ARES rheometer (advanced rheometric expansion system, Rheometric Scientific Inc.). A rheological frequency sweep from 0.0628 to $100 \mathrm{rad} / \mathrm{s}$ was performed with a shear strain of $0.5 \%$ in the parallel-plate geometry with a temperature range of $0-90^{\circ} \mathrm{C}$. The disc-shaped samples with thicknesses of $\sim 1 \mathrm{~mm}$ and diameters of $15 \mathrm{~mm}$ were adhered to the plates with superglue (Aron alpha, Konishi, Japan) and surrounded by water to maintain hydration.

Scanning electron microscopy (SEM). The structure of the gels was observed by SEM (JSM-6010LA, JEOL, JAPAN) with an accelerating voltage of $20 \mathrm{kV}$. The sample gel specimens were prepared by freeze-drying the swollen gel to avoid shrinkage in the drying process. The sample was cryogenically fractured in liquid nitrogen. Before SEM characterization, the surface was treated with a thin layer of gold by sputtering method.

GPC Analysis. Molecular weights $\left(M_{\mathrm{w}}\right)$ of PMPTC were determined by GPC measurement using a HITACHI L-7110 pump and a HITACHI 7490 RI detector, using a method the same as in a previous study ${ }^{34}$ with a slight modification: a buffer solution of $0.3 \mathrm{M} \mathrm{Na}_{2} \mathrm{SO}_{4}$ was used as an eluent at $40{ }^{\circ} \mathrm{C}$, and flow rate was $0.4 \mathrm{~mL} / \mathrm{min}$. 


\section{Results and discussion}

\subsection{Effects of charge fraction, monomer concentration, and molecular weight on the properties of polyion-complex hydrogels}

\subsubsection{Charge fraction}

Polyion-complex (PIC) hydrogels are crosslinked by inter-polymer ionic bonds, and therefore the charge ratio in feed between the two oppositely charged monomers dramatically influences the resulting polymer properties. Figure 1 presents the effect of the in feed molar fraction of the second monomer NaSS, $f$, on the swelling volume ratio, $Q_{v}$, and the work of extension, $W_{b}$, of the PMPTC/PNaSS(1.5-f-0.05-0.1) PIC hydrogels. In a relatively wide composition range $(f=0.45 \sim 0.55)$ investigated, stable physical gels are formed without the presence of any chemical cross-linker. Near the charge balance point $(f=0.49 \sim 0.52)$, the gels shrink in water $\left(Q_{v}<1\right)$ relative to their as-prepared state, indicating that the Coulombic attraction between chains prevails over the repulsion between monomer units and the polymer chains collapse. In regions with sufficient charge imbalance $(f<0.49$ or $f>0.52)$, on the other hand, the gels swell $\left(Q_{v}>1\right)$, indicating that the Coulombic repulsion prevails and the unpaired ion-contain segments preferentially interact with water, resulting in polymer elongation. The shrinking of the gels around the charge balance point is accompanied by a dramatic increase in the work of extension, $W_{b}$, while the swelling samples are very weak (data not shown). Thus, the optimized $f$ value of the feed, at which $Q_{v}$ reaches a minimum and $W_{b}$ reaches a maximum, is almost at 0.5 , which is the charge balance point. The true charge ratio, $f_{\text {true }}$, of PMPTC/PNaSS(1.5-f-0.05-0.1) was studied using elemental analysis (Table 1). The results revealed that best molar fraction $f=0.5$ in feed also yields $f_{\text {true }}=0.5$, which is at 
the ideal stoichiometric ratio of $1: 1$. It indicates that in this PIC hydrogel, complete charge balance $\left(f_{\text {true }}=0.5\right)$ results in the most stable state.

In the case of PA system, however, stable physical gels can only be formed around the charge balanced composition $(f \sim 0.5)$. Deviation from this composition gives extremely weak gels or polymer solution. For comparison, we show the behaviors of chemically crosslinked PA hydrogels $\mathrm{P}(\mathrm{MPTC}-\mathrm{co}-\mathrm{NaSS})$ 0.875-f-4 obtained from the previous work in Figure 1b. ${ }^{28,33}$ The PA hydrogels also present the largest shrinkage and highest work of extension at the ideal stoichiometric ratio. In the following work, we focus our study on the physical PIC and PA hydrogels with charge balanced composition $f=0.5$.

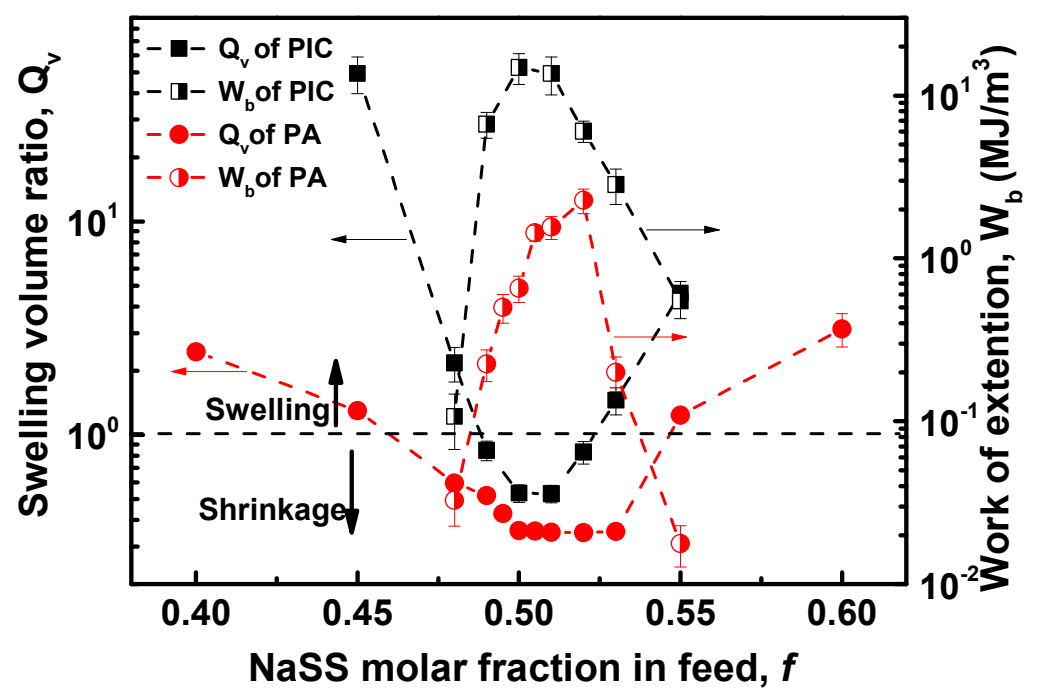

Figure 1. NaSS molar fraction $(f)$ effects on the swelling volume ratio in relative to the as-prepared state, $Q_{v}$, and the work of extension, $W_{b}$, of the PIC hydrogels PMPTC/PNaSS(1.5-f-0.05-0.1). Without chemical crosslinking, the PA system can only form physical gels near the charge balanced composition $(f \sim 0.5)$. For comparison, the behaviors of chemically crosslinked PA hydrogels P(MPTC-co-NaSS) $0.875-f-4$ are also shown. The data of P(MPTC-co-NaSS) hydrogels are cited from ref. 28 
Table 1 The weight percentage of elements in various PIC hydrogels PMPTC/PNaSS(1.5-f-0.05-0.1) determined by element analysis.

\begin{tabular}{ccccccc}
\hline$f^{*}$ & $\mathrm{C}(\mathrm{wt} \%)$ & $\mathrm{H}(\mathrm{wt} \%)$ & $\mathrm{N}(\mathrm{wt} \%)$ & $\mathrm{S}(\mathrm{wt} \%)$ & $\mathrm{Cl}(\mathrm{wt} \%)$ & $f_{\text {true }}{ }^{* * *}$ \\
\hline 0.49 & 54.73 & 7.97 & 7.42 & 7.79 & 0.32 & 0.478 \\
0.50 & 55.89 & 8.04 & 7.27 & 8.33 & 0 & 0.500 \\
0.51 & 55.59 & 7.87 & 7.11 & 8.47 & 0 & 0.510 \\
0.52 & 55.63 & 7.87 & 7.07 & 8.55 & 0 & 0.514 \\
\hline
\end{tabular}

\subsubsection{Effect of monomer concentration}

The mechanical properties of the PIC gels are strongly related to the concentration of monomer during preparation. Figure 2a shows the images of as-prepared PIC samples of PMPTC/PNaSS $\left(C_{m}-0.5-0.05-0.1\right)$ polymerized with different total monomer concentration, $C_{m}$, from $0.2 \mathrm{M}$ to $2.2 \mathrm{M}$. All of the as-prepared samples are a white, opaque gel. When the $C_{m}$ is as low as $0.2 \mathrm{M}$, a thin free water layer appears on the surface of the gel. This indicates that the lowest monomer concentration required to form a nonphase-separated gel is around $0.2 \mathrm{M}$. In contrast, when the $C_{m}$ is above $2.2 \mathrm{M}$, the viscosity of the mixture at the second polymerization stage becomes too high to dissolve the components.

As shown in Figure 2b, when these as-prepared PIC gels are dialyzed in water, they all shrink. The lower the in feed monomer concentration $C_{m}$, the larger the shrinkage. The shrinking volume ratio, $\mathrm{Q}_{\mathrm{v}}$, changes from 0.1 to 0.8 when $C_{\mathrm{m}}$ increases from $0.2 \mathrm{M}$ to 2.2 
M. These shrinkage behaviors of PIC hydrogels with respect to $C_{m}$ are very different from that of the PA hydrogels. The PA samples of P(MPTC-co-NaSS) $\left(C_{m}-0.52\right)$ show the low critical concentration for forming hydrogel at $C_{m}=1.3 \mathrm{M}$, much higher than that of PIC system of $0.2 \mathrm{M}^{28}$ Furthermore, the PA gels swell $\left(Q_{v}>1\right)$ at $C_{m}<1.6 \mathrm{M}$ and shrink $\left(Q_{v}<1\right)$ at $C_{m}>1.6 \mathrm{M}$, reaching a constant $Q_{v}$ value at high $C_{m}$, as also shown in Figure $2 \mathrm{~b} .^{28,33}$. It is interesting to observe that the $Q_{v}$ values of PIC and PA almost overlap at high $C_{m}$ region.

On the basis of $C_{m}$ and $Q_{v}$, the resulting polymer concentration at equilibrium can be estimated from $C_{p}=C_{m} / Q_{v}$. In the case of PIC, $C_{p}$ is approximately constant of $2.5 \mathrm{M}$, independent of the initial monomer concentration in feed $C_{m}$, as shown in Figure $2 \mathrm{~b}$. The water content measurement also confirmed that PIC samples of $C_{m}$ from $0.2 \mathrm{M}$ to $2.2 \mathrm{M}$ have the almost identical water content around 50 55 wt $\%$. On the other hand, the $C_{p}$ of PA samples gradually increases with $C_{m}$ and reaches $2.9 \mathrm{M}$ at high $C_{m}=2.2 \mathrm{M}$, corresponding to a water content of $60 \mathrm{wt} \%$. It is surprise, that the water content of the PIC gels (50 55 wt\%) is very close to but slightly lower than that of PA gels prepared at high monomer concentration $\left(C_{m}=2.2 \mathrm{M}\right) .{ }^{28,33}$ This indicates that the overall ionic bonds packing density of the PIC system is independent of the $C_{m}$ and is slightly lower than the PA prepared at high concentration.

The correlations between the modulus $E$ measured after dialysis and $C_{m}$ are shown in Figure 2c. The PIC samples, having the constant $C_{p}$, show almost constant modulus, $E$, for $C_{m}=0.2 \mathrm{M} \sim 1.5 \mathrm{M}$, and the E slightly decreases when $C_{m}>1.5 \mathrm{M}$ (Figure 2c). On the other hand, the PA samples, of which $C_{p}$ increases with $C_{m}$, show increases of $E$ with $C_{m}$ and then the $E$ saturates to the value of PIC at $C_{m}>1.6 \mathrm{M}$. 
The above results indicate that PIC system have stronger ionic bond forming ability than the PA system. The different $C_{m}$ effects on the volume change $Q_{v}$ and therefore on the polymer concentration $C_{p}$ of the gels for PIC and PA systems should be attributed to their differences in polymer chain conformation. For the PIC system, the polymer chains, carrying homo-charges, are in relatively extended conformation before complexation, which favors the inter-chain ion bonds formation even at relatively low concentration; while the PA chains, carrying opposite charges in each single chain, are in compact conformation due to intra-chain ionic bond formation, which requires very high concentration to form inter-chain bonding. This explains why the PIC system has a much lower critical concentration to form supramolecular gels than that of the PA system.

Even though the polymer concentration $C_{p}$ and modulus $E$ show weak dependence on the preparation concentration $C_{m}$, the tensile properties of PIC gels do show a strong dependence on $C_{m}$ as shown in Figure 2d. In similar to the modulus, the yielding stress are also almost the same for $C_{\mathrm{m}}=0.2 \sim 1.5 \mathrm{M}$, but it becomes small when $C_{\mathrm{m}}>1.5 \mathrm{M}$. The differences in initial monomer concentration also induce significant distinction in elongation at break (Figure 2d). When $C_{\mathrm{m}}<0.7 \mathrm{M}$, the samples are relatively weak and fail under low strain and stress. The elongation at break of the sample with $C_{\mathrm{m}}=0.2$ is only 1.8 , while it is up to 9.0 for the sample with $C_{\mathrm{m}}=2.0$. The $C_{m}$ dependence of the fracture strain indicates the effect of trapped topological entanglement on the tensile behaviors. Generally, the entanglement density of the polymer chains increases with the concentration in a power law relation as $\sim C_{m}^{2.3} \cdot 35$ The low concentration $\left(C_{m}<0.7 \mathrm{M}\right)$ results in weak entanglement, and therefore low strain-at-break when undergoing extension. When $C_{m} \geq 0.7 \mathrm{M}$, the fracture strain becomes large and does not show 
systematic dependence on $C_{m}$, which indicates that the polymer chains have strong entanglement to sustain large elongation, and the fracture is due to breaking of covalent bonds. The slight decrease in Young's modulus and yielding stress of samples, and slight increase in the fracture strain for samples prepared at $C_{m}>1.5 \mathrm{M}$ suggest that at extremely high concentration the ionic interaction is weakened, probably by self-screen effect.

Therefore, for the PIC hydrogels, the work of extension, $W_{b}$, a quantity for assessment of toughness, increases with $C_{m}$ at $C_{m}<0.7 \mathrm{M}$ and the largest values of $W_{b}$ are obtained for samples with $C_{m}$ ranging from $0.7 \mathrm{M}$ to $1.5 \mathrm{M} ; W_{b}$ slightly decreases with increasing $C_{m}$ when $C_{m}>1.5 \mathrm{M}$ (Figure 2e). Particularly, the $W_{b}$ increases from $1.9 \mathrm{MJ} / \mathrm{m}^{3}$ to 14.6 $\mathrm{MJ} / \mathrm{m}^{3}$ for $C_{m}=0.2$ and $C_{m}=1.6$, respectively. In contrast, the PA samples monotonously increase the mechanical toughness $W_{b}$ with the increase in $C_{m}$, indicating that the entanglement effect dominates the tensile behaviors for the whole $C_{m}$ range investigated. 28,33 At the optimized condition, the PIC gels show about two times of yielding stress, fracture stress but similar fracture strain in comparison with those of PA gels prepared at the highest concentration investigated $C_{m}=2.2 \mathrm{M} .{ }^{28,33}$ Thus, $W_{b}$ of optimized PIC is about two times of that of PA $\left(C_{m}=2.2 \mathrm{M}\right)$ as shown in Figure $2 \mathrm{e}$.

(a)

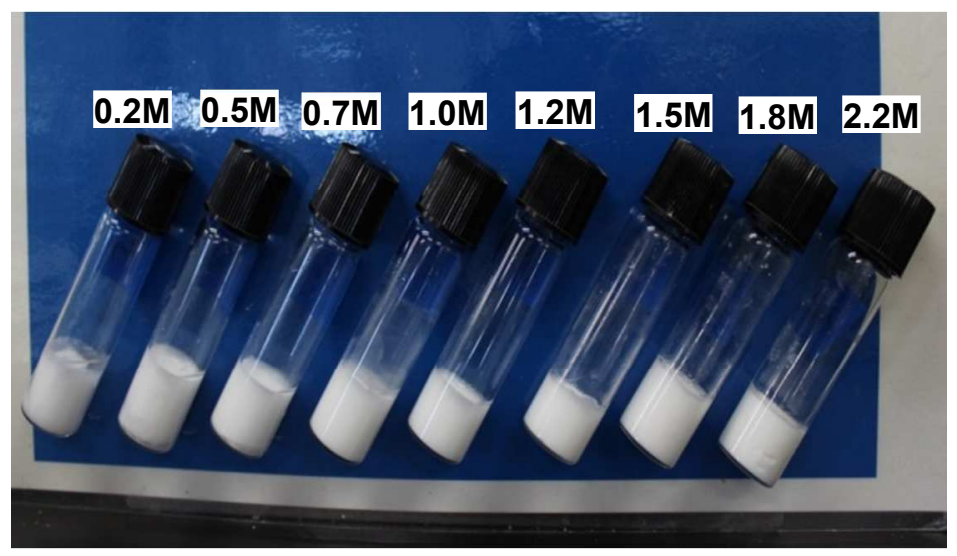


(b)

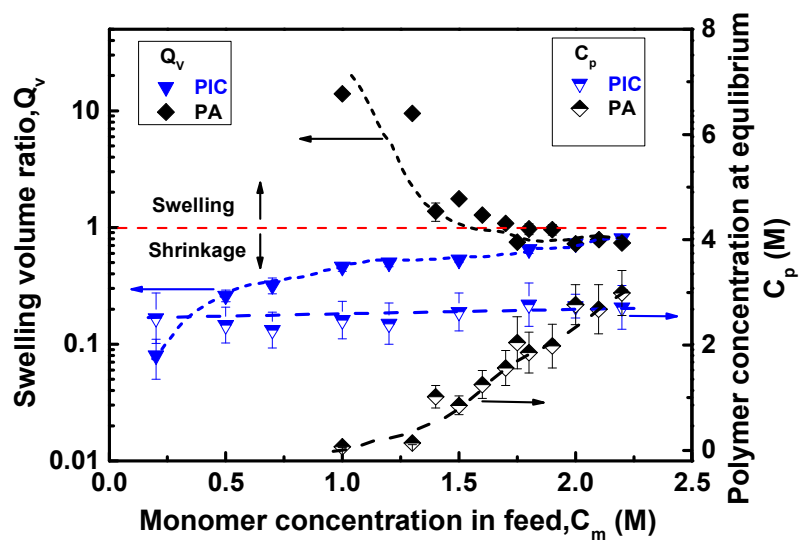

(d)

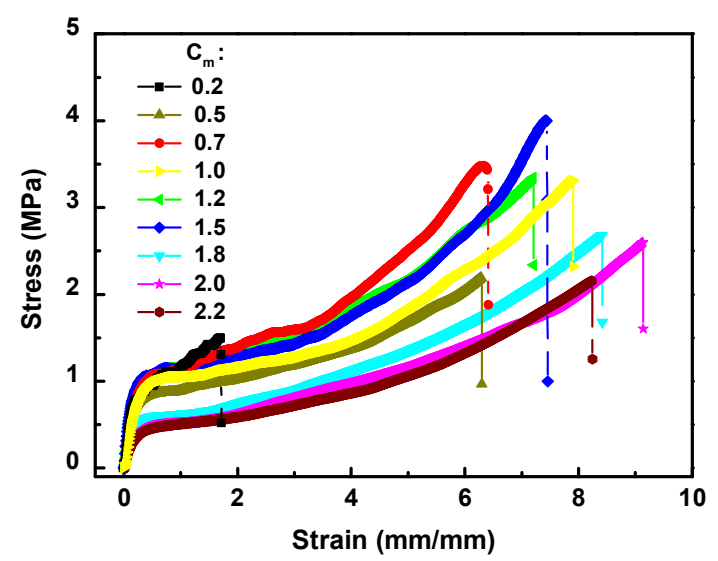

(c)

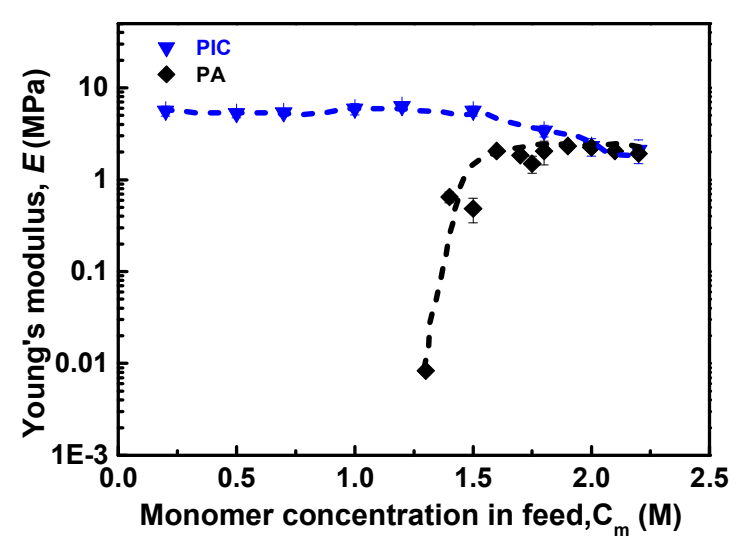

(e)

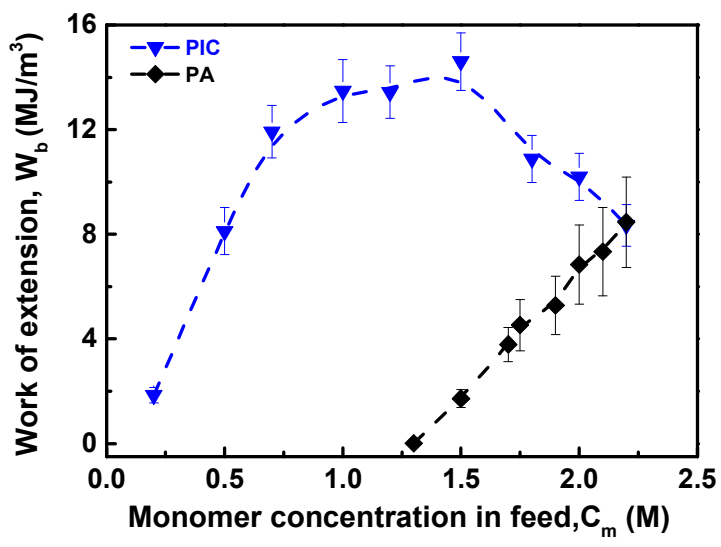

Figure 2. Effect of monomer concentration on the physical properties of PIC hydrogels.

(a) Photographs of the PMPTC/PNaSS $\left(C_{\mathrm{m}}-0.5-0.05-0.1\right)$ hydrogels polymerized with different total monomer concentration in feed, $C_{\mathrm{m}}$. Data in the figure are $C_{\mathrm{m}}$ in unit of $\mathrm{M}$. (b) The $C_{\mathrm{m}}$ dependence of the volume ratio, $Q_{v}$, of the dialysed sample to the as-prepared sample, and of the polymer concentration, $C_{p}=C_{\mathrm{m}} / Q_{v}$, of the dialysed sample. (c) The $C_{\mathrm{m}}$ dependence of Young's modulus $(E)$. (d) Representative stress-strain curves of PIC hydrogels with different $C_{m}$. (e) The $C_{\mathrm{m}}$ dependence of work of extension, $W_{b}$. For comparison, the behaviors of PA hydrogels $\mathrm{P}(\mathrm{MPTC}-\mathrm{co}-\mathrm{NaSS})\left(C_{\mathrm{m}}-0.52\right)$ are also shown in (b), (c), (e). Both PIC and PA hydrogels were prepared at the compositions to form 
charge balanced samples. The data of PA hydrogels P(MPTC-co-NaSS) are cited from ref. 28 and ref. 33 .

\subsubsection{Molecular weight}

The molecular weight of PMPTC of the PIC hydrogels discussed in the above section was measured as $\sim 10^{6} \mathrm{~g} / \mathrm{mol}$ (Table 2), approximately equivalent to that of copolymer in PA hydrogel (around $\sim 10^{6} \mathrm{~g} / \mathrm{mol}$ ) in our previous report. ${ }^{28}$ For radical polymerization, the molecular weight is strongly dependent on the initiator concentration for polymerization at fixed monomer concentrations. As shown in Table 2, the molecular weight of the PMPTC decreases with increasing initiator concentration in monomer solution. Specifically, the $\mathrm{M}_{\mathrm{w}}$ of PMPTC polymerized by $0.05 \mathrm{~mol} \%$ initiator is 3 times of that of PMPTC polymerized by $0.5 \mathrm{~mol} \%$ initiator. As PNaSS polymerized at the second step was tightly bonded with the PMPTC by ionic bonds, we could not measure the molecular weight of the PNaSS in the PIC gels. The molecular weight of PNaSS should also decrease with increasing initiator concentration. The molecular weight effect of the PMPTC and PNaSS on the mechanical properties of the PIC hydrogels is studied. Figure 3a shows that the PIC hydrogels, PMPTC/PNaSS(1.5-0.5- $\left.x_{1}-0.1\right)$, composed of PMPTC polymerized with low initiator concentration have higher yielding stress and fracture energy than that of the hydrogels composed of PMPTC polymerized with high initiator concentration, while the fracture strain is almost independent of the initiator concentration. Similar results are observed for the PIC hydrogels, PMPTC/PNaSS(1.50.5-0.05- $x_{2}$ ), composed of PNaSS synthesized with different initiator concentrations $x_{2}$ $(\mathrm{mol} \%)$ at the second step of polymerization, as shown in Figure 3b. Combining the 
results of Table 2 and Figure 3, we know that the higher the molecular weight of the polymers, the higher the modulus, the yielding stress, and the toughness of the PIC hydrogels, either for the first or the second polymers. Since the yielding stress and fracture stress depend on the molecular weight while the fracture strain does not, this molecular weight effect suggests that the ionic bonds are stabilized by the increased entanglement. This phenomenon is similar to the case of adding the chemical crosslinker, which also stabilizes the ionic bonds. ${ }^{30}$

Table 2. Molecular weight of PMPTC- $x_{1}$ measured by GPC. Here, $x_{1}(\mathrm{~mol} \%)$ is initiator concentration relative to the monomer MPTC.

\begin{tabular}{cccc}
\hline PMPTC- $x_{1}$ & Mn $(\mathrm{g} / \mathrm{mol})$ & Mw $(\mathrm{g} / \mathrm{mol})$ & Mw/Mn \\
\hline PMPTC-0.05 & $3.75 \times 10^{6}$ & $4.66 \times 10^{6}$ & 1.24 \\
PMPTC-0.1 & $9.45 \times 10^{5}$ & $2.11 \times 10^{6}$ & 2.24 \\
PMPTC-0.5 & $5.81 \times 10^{5}$ & $1.49 \times 10^{6}$ & 2.57 \\
\hline
\end{tabular}

(a)

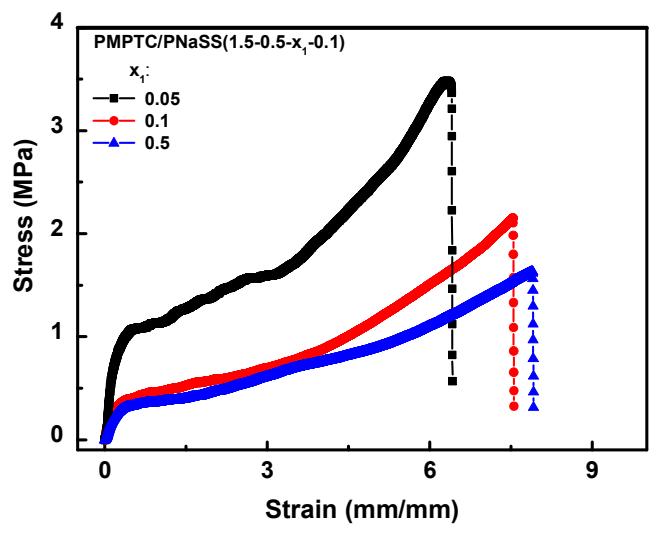

(b)

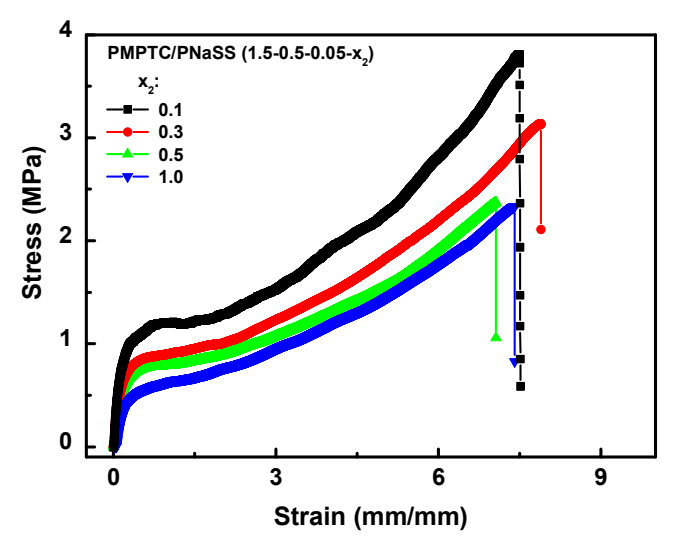

Figure 3. (a) Stress-strain curves of PMPTC/PNaSS(1.5-0.5- $\left.x_{1}-0.1\right)$ hydrogels with different initiator concentrations $x_{1}(\mathrm{~mol} \%)$ at the first step of polymerization. (b) Stress- 
strain curves of PMPTC/PNaSS(1.5-0.5-0.05- $\left.x_{2}\right)$ hydrogels with different initiator concentrations $x_{2}(\mathrm{~mol} \%)$ at the second step of polymerization.

\subsection{Structure analysis of PIC hydrogels by rheology}

We have analysed the structure of the PIC hydrogels by rheology that is an effective way to study the morphology of the system. Figure $4 \mathrm{a}$ shows the result of a dynamic temperature sweep for PMPTC/PNaSS(1.5-0.5-0.05-0.1). The thermal energy causes the rupture of ion bonds and increases the dynamics of polymer segments, therefore, the storage modulus $\left(G^{\prime}\right)$ and the loss modulus $\left(G^{\prime \prime}\right)$ decrease about two orders of magnitude upon heating from $0{ }^{\circ} \mathrm{C}$ to $75^{\circ} \mathrm{C}$ and then level off above $75^{\circ} \mathrm{C}$. The tan $\delta$ curve exhibits a main peak at $51{ }^{\circ} \mathrm{C}$ and a broad shoulder peak at $27{ }^{\circ} \mathrm{C}$, suggesting that some micro phase separation structure might exist in the PIC hydrogel to form the ionic bonds of different strength. For samples at $C_{\mathrm{m}}=1.2 \mathrm{M}$ and $2.0 \mathrm{M}$, the $G^{\prime}$ and $G^{\prime \prime}$ also decrease with increasing temperature which are similar to the tendency of $C_{\mathrm{m}}=1.5 \mathrm{M}$ (curves are not shown). With increasing monomer concentration $C_{m}$, the intensity of $\tan \delta$ decreases, and the main peak of $\tan \delta$ shifts to lower temperature, as shown in Figure 4b. For hydrogels synthesized from $C_{\mathrm{m}}=2.0 \mathrm{M}$, the peak at low temperature becomes the main peak. This indicates that at high concentrations that poor dispersion occurs, the formation of relatively strong ion bonds is suppressed, resulting in a majority of weak bonds. This result is in agreement with the decrease of the modulus when $C_{\mathrm{m}}>1.5 \mathrm{M}$ (Figure 2c).

The dynamic behaviors of the PIC hydrogels at different temperatures and frequencies well follow the principle of time-temperature superposition. Figure 4c shows the master curves of $G^{\prime}, G^{\prime \prime}$, and $\tan \delta$ for the PMPTC/PNaSS(1.5-0.5-0.05-0.1) hydrogel at a 
reference temperature of $20{ }^{\circ} \mathrm{C}$. For comparison, the corresponding master curves of P(MPTC-co-NaSS) (2.1-0.52) hydrogel are also shown in Figure 4c. For both PIC gel and PA gel, $G^{\prime}$ is larger than $G^{\prime \prime}$ over the whole frequency range from $10^{-7}$ to $10^{6} \mathrm{rad} / \mathrm{s}$, indicating that both gels, even without any chemical cross-linking, are always in the gel state with predominantly elastic properties. The PIC gel shows a main peak and a broad sub-peak at frequencies around $5 \times 10^{-3} \mathrm{rad} / \mathrm{s}$ and $1 \mathrm{rad} / \mathrm{s}$, respectively, in its $\tan \delta$ relaxation curve. This result agrees with the results of the temperature sweep that suggest the micro phase separated structure of PIC hydrogels to give a wide range of ion bond strengths. On the other hand, only one broad single peak appears in the $\tan \delta$ curve of PA hydrogel at the frequency $10^{-2} \mathrm{rad} / \mathrm{s}$, in between the two frequencies for the two $\tan \delta$ peaks of PIC hydrogel, indicating that there is no distinct phase separation of PA hydrogel. The $\tan \delta$ value of PIC hydrogel is higher than that of PA hydrogel over the wide frequency range except at the high frequency end, indicating the relatively stronger viscoelasticity of the PIC system than that of the PA system.

The apparent activation energy $E_{a}$ of ionic bonds can be determined using the Arrhenius equation, $a_{T}=A e^{E_{a} / R T}$, where $a_{T}$ is the shift factor, $R$ is the ideal gas constant, and $A$ is a constant. ${ }^{7}$ As shown in Figure 4d, the Arrhenius plot of the PIC gel shows two linear regions with a reflection point at c.a. $50{ }^{\circ} \mathrm{C}$, giving two activation energies $E_{a}: 62 \mathrm{~kJ} / \mathrm{mol}$ at high temperature region and $302 \mathrm{~kJ} / \mathrm{mol}$ at low temperature region, corresponding to $25 k_{B} T$ and $121 k_{B} T$ (in relative to the room temperature), respectively. The activation energy at low temperature is less than, but close to, the covalent bond dissociation energy, $E_{c-c} \sim 347 \mathrm{~kJ} / \mathrm{mol}\left(\sim 140 k_{B} T\right)$. This explains why a rigid and tough gel is observed even without any covalent cross-linker below the softening temperature. The shift factor $a_{T}$ 
behaviours of the PIC system is very similar to that of the PA hydrogels that also show two activation energies, $71 \mathrm{~kJ} / \mathrm{mol}$ and $308 \mathrm{~kJ} / \mathrm{mol}$ above and below the softening temperature. ${ }^{28}$ Although the primary structure of the PIC hydrogels and PA hydrogels are completely different, the quite similar shift factor $a_{T}$ of the two systems suggests that the ionic bond strengths at short molecular scale are similar. Accordingly, the difference of the mechanical behaviors of the two systems comes from the difference in more large scale structure. This is reasonable since both inter-chain and intra-chain bonding exist in PA gels but only inter-chain bonding exists in PIC gels. The latter should have more topological entanglement.

(a)

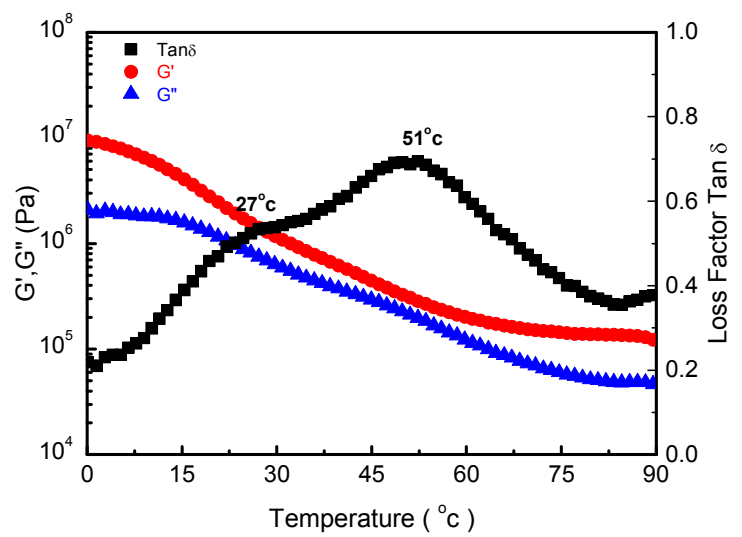

(c)

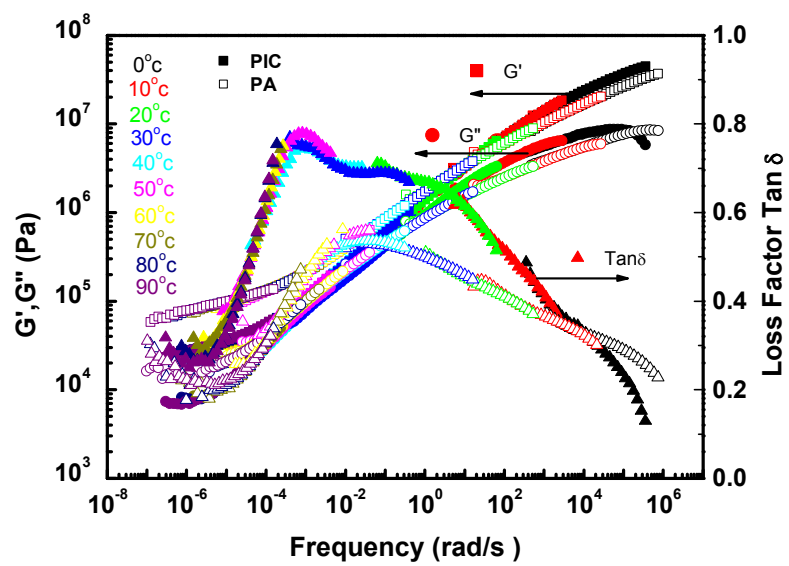

(b)

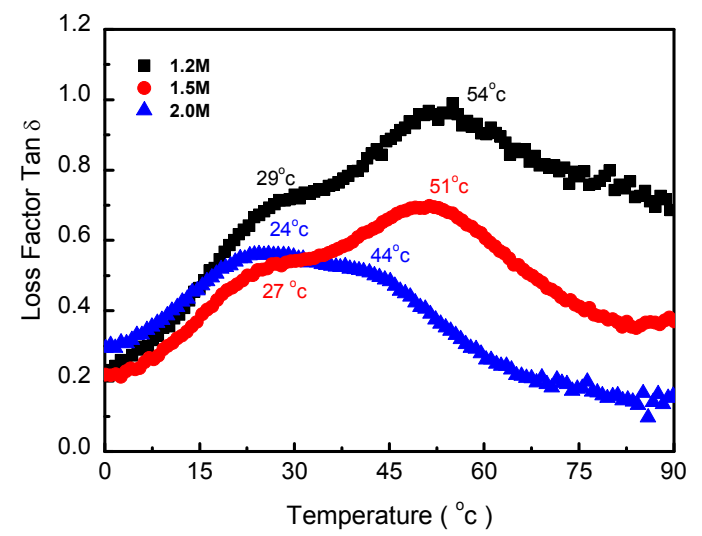

(d)

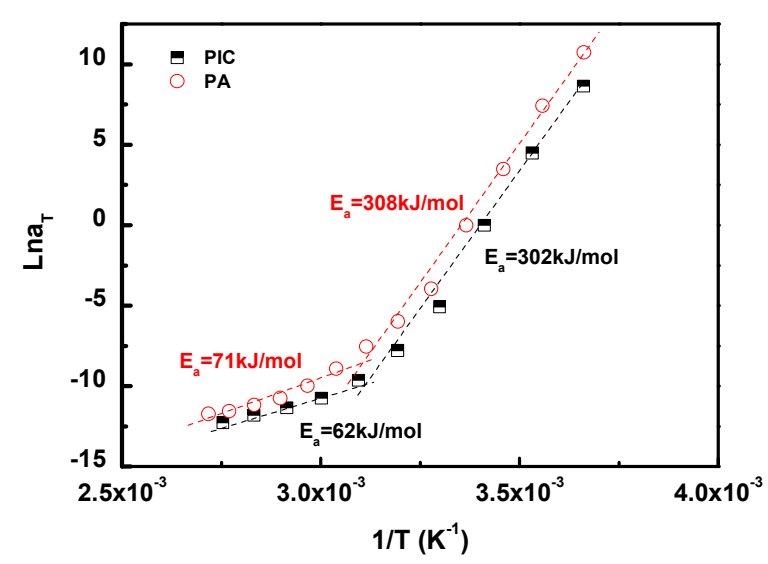


Figure 4. Dynamic mechanical behaviours of the PIC hydrogels in comparison with PA hydrogels. (a) Temperature dependence of storage modulus, $G^{\prime}$, loss modulus, $G^{\prime \prime}$, and loss factor, $\tan \delta$, of PMPTC/ PNaSS(1.5-0.5-0.05-0.1) hydrogel at $6.28 \mathrm{rad} / \mathrm{s}$ and $0.1 \%$ strain. A main peak and a shoulder peak appear in the $\tan \delta$ curve corresponding to two softening temperatures, $T_{s}$, which are indicated by the numbers in the figure; (b) comparison of $\tan \delta$ for samples $\mathrm{PMPTC} / \mathrm{PNaSS}\left(C_{m^{-}}-0.5-0.05-0.1\right)$ prepared at different monomer concentration, $C_{m}$. (c) Frequency $(\omega)$ dependences of the storage modulus, $G^{\prime}$, loss modulus, G", and loss factor, $\tan \delta$, of PMPTC/PNaSS(1.5-0.5-0.05-0.1) hydrogel (filled symbols) and P(MPTC-co-NaSS) (2.1-0.52) hydrogel (open symbols). The measurements were performed from 0.0628 to $100 \mathrm{rad} / \mathrm{s}$ at a shear strain of $0.5 \%$ at different temperatures from $0{ }^{\circ} \mathrm{C}$ to $90^{\circ} \mathrm{C}$, and the results were obtained by performing classical time-temperature superposition shifts at a reference temperature of $20{ }^{\circ} \mathrm{C}$; (d) Arrhenius plot depicting the temperature dependence of the shift factors for the samples. The apparent activation energy values, $E_{a}$, were calculated from the slopes of the curves. The data of P(MPTC-co-NaSS) hydrogel are cited from ref. 28.

\subsection{Structure Analysis by Scanning Electron Microscopy}

To further explore the structure, the structure of freeze-dried PIC and PA hydrogels are observed by SEM and the results are shown in Figure 5. For PIC hydrogel, it exhibits large segregated structure with large pores of diameter ranging from 0.5 to $3 \mu \mathrm{m}$. Moreover, the dehydrated polymer formed a granular shape with size around $0.5 \mu \mathrm{m}$, suggesting that many chains tightly pack to form a globular structure via the inter-chain ionic bonds. On the other hand, PA hydrogel shows more homogenous structure with 
network of a relatively smaller size ranging from 0.1 to $0.3 \mu \mathrm{m}$. These results are in good agreement with the rheological measurements that show two relaxation peaks of the PIC hydrogels and single but broad relaxation peak for PA hydrogels.
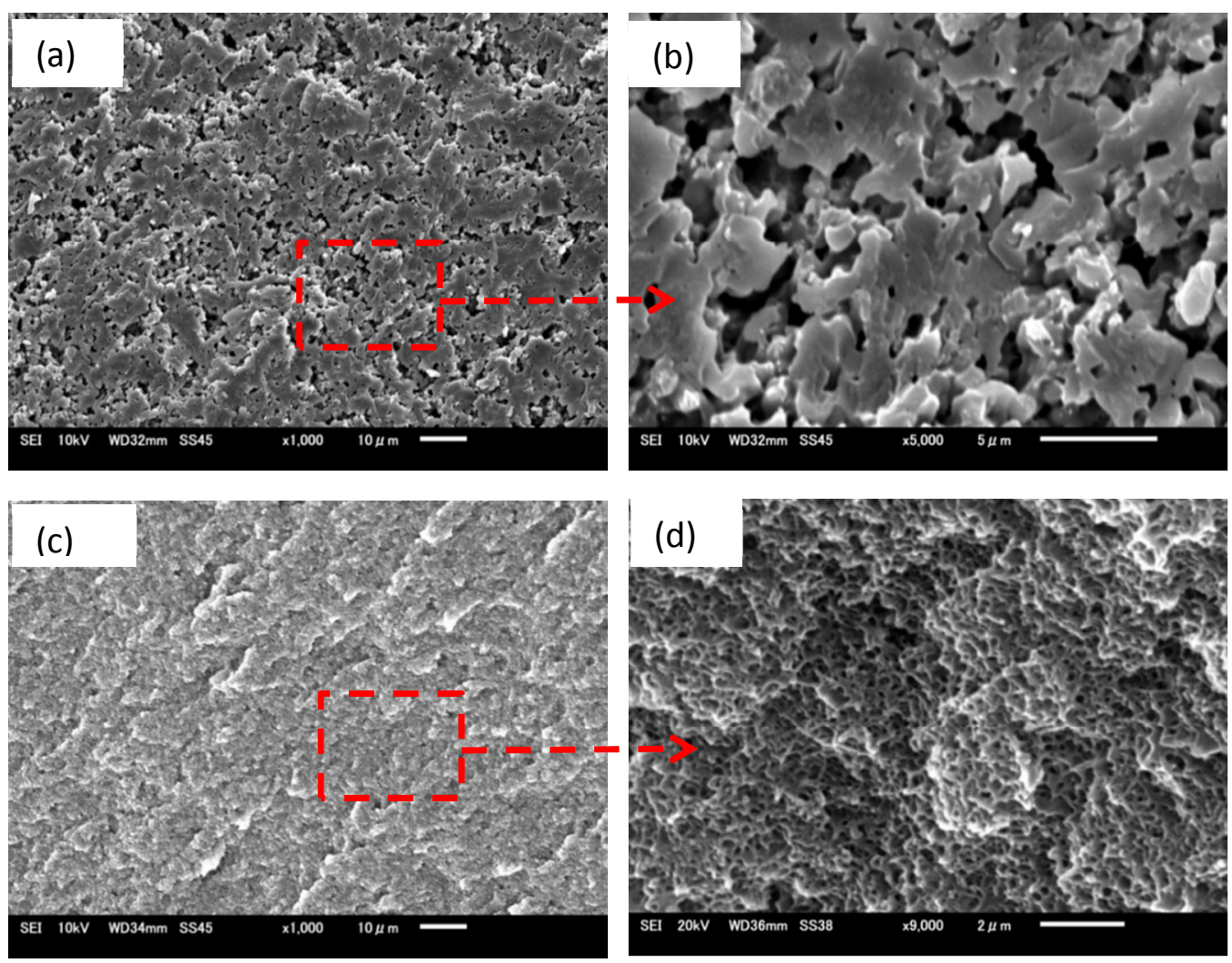

Figure 5. SEM photographs of microstructure of the PIC and PA hydrogels. (a) PMPTC/PNaSS (1.5-0.5-0.05-0.1) hydrogel; (b) The magnification of the zone in (a); (c) P(MPTC-co-NaSS) (2.0-0.52) hydrogel; (d) The magnification of the zone in (c).

\subsection{Viscoelastic behaviors at large deformation}

To ascertain the nonlinear and viscoelastic behavior of the PIC hydrogels at large deformation, we performed uniaxial stretching experiments at various strain rates for the sample of PMPTC/PNaSS (1.5-0.51-0.05-0.1). The tensile stress-strain curves (nominal stress, $\sigma$, vs nominal strain, $\varepsilon$ ) are plotted in Figure 6a. The corresponding data from the 
curves are listed in Table 3. The mechanical properties are strongly influenced by strain rate. Yielding is clearly observed around $\varepsilon=0.5$, and the yield stress, $\sigma_{y}$, increases from $0.08 \mathrm{MPa}$ to $0.69 \mathrm{MPa}$, as the strain rate increases from 0.0014 to $0.14 \mathrm{~s}^{-1}$. The fracture stress, $\sigma_{b}$, also increases with the strain rate from 0.47 MPa to $3.2 \mathrm{MPa}$, while the fracture strain decreases slightly. The Young's modulus, $E$, of samples notably increases about 15 times from $0.34 \mathrm{MPa}$ at $0.0014 \mathrm{~s}^{-1}$ to $5.13 \mathrm{MPa}$ at $0.14 \mathrm{~s}^{-1}$. We can see that the strain ratedependent property for PIC hydrogels are similar to that of PA hydrogels. ${ }^{29}$

According to a recent report by Mayumi et al., the effects of strain and time can be separated for a polyvinyl alcohol (PVA) hydrogel with dual cross-linking, one is permanent, chemical crosslinking, and the other is reversible, physical crosslinking. ${ }^{36}$ Similar strain-time separation was observed for the PA hydrogels at low strain rate. ${ }^{29}$ Here, we also plot the reduced stress, $\sigma^{*}=\sigma /\left(\lambda-\lambda^{-2}\right)$, against the stretching time, $t$, where $\lambda=\varepsilon+1$ is the stretching ratio. As shown in Figure $6 \mathrm{~b}, \sigma^{*}(t)$ shows a decreasing trend with $t$ except for the strain hardening regimes where the reduced stress increases rapidly with time. This indicates that the physical PIC hydrogels behaves as dually cross-linked hydrogels, containing permanent cross-linking (strong bonds) and breakable cross-linking (weak bonds) during deformation. The $\sigma^{*}(t) \sim \mathrm{t}$ curves measured at relatively low stretching rate $\left(0.0014 \mathrm{~s}^{-1}, 0.007 \mathrm{~s}^{-1}\right)$ overlap, exhibiting a master curve with a slope close to -0.6. This indicates that the strain-time separation is also applicable to PIC hydrogels at low stretching rate, and strain/strain rate have no influence on the breakage rate of the reversible weak bonds. However, at high strain rate $\left(0.014 \mathrm{~s}^{-1}\right.$ or higher $)$, the $\sigma^{*}(t) \sim \mathrm{t}$ curves deviate below the master curve, indicates that the high strain rate accelerate the breakage rate of the weak bonds to give a low stress. The strain rate limit of $0.007 \mathrm{~s}^{-1}$ for 
holding the strain-time separation principle is in reasonable agreement with one of the two relaxation peaks at low frequency side $\left(5 \times 10^{-3} \mathrm{rad} / \mathrm{s}\right)$ in Figure $4 \mathrm{c}$. This indicates that the strain-time separation principle is hold when the stretching rate is slower than the inverse of the weak bond breaking time.

(a)

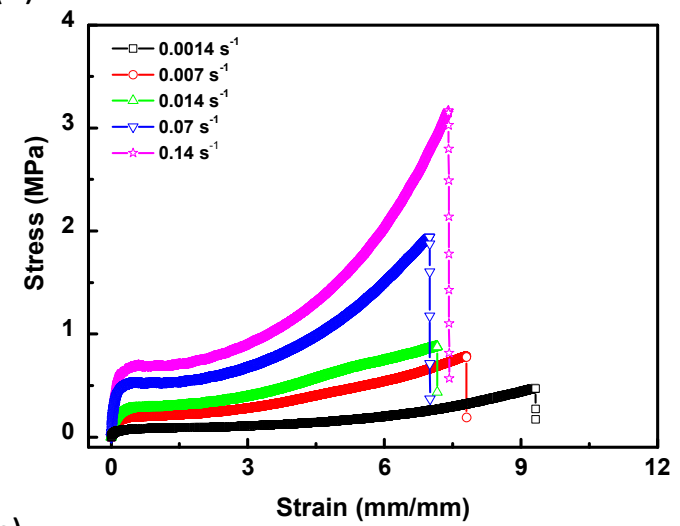

(c)

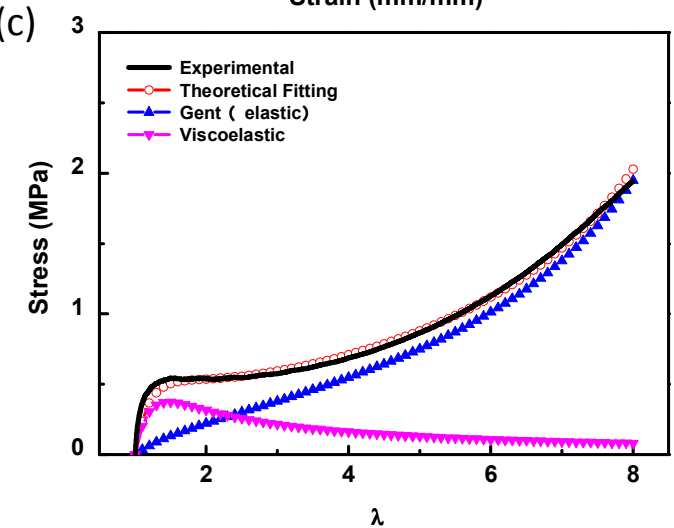

(b)

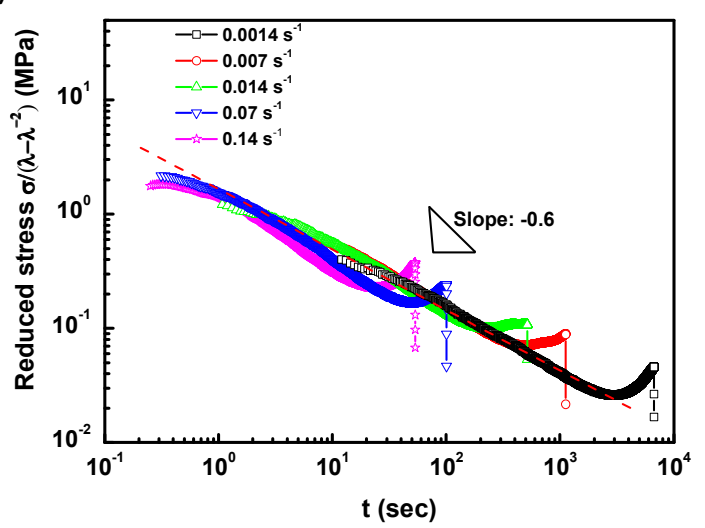

Figure 6. (a) Tensile stress-strain curves for the PMPTC/PNaSS(1.5-0.51-0.05-0.1) hydrogel at various strain rates; (b) Time dependence of the reduced stress $\sigma^{*}=\sigma /\left(\lambda-\lambda^{-2}\right)$ at various strain rates, the slope of dash line is -0.6 ; (c) Typical tensile behaviour at 0.07 $\mathrm{s}^{-1}$ (experimental data), and simulated sum of contribution (theoretical fitting curve), and their individually contributions from the viscoelastic part and elastic part (Gent). 
Table 3. Summary of the tensile test properties of PMPTC/PNaSS(1.5-0.51-0.05-0.1) hydrogel measured at various stretch rates.

\begin{tabular}{ccccccccccc}
\hline $\begin{array}{c}\text { Stretch rate } \\
\left(\mathrm{s}^{-1}\right)\end{array}$ & $\begin{array}{c}E \\
(\mathrm{MPa})^{*}\end{array}$ & $\begin{array}{c}\varepsilon_{\mathrm{b}} \\
(\mathrm{MPa})\end{array}$ & $\begin{array}{c}\sigma_{y} \\
(\mathrm{MPa})\end{array}$ & $\begin{array}{c}\sigma_{\mathrm{b}} \\
(\mathrm{MPa})\end{array}$ & $G_{v}$ & $D_{\mathrm{e}}$ & $G_{\mathrm{e}}$ & $J_{\mathrm{m}}$ & $\lambda_{\mathrm{m}}$ & $G_{\mathrm{v}} / G_{\mathrm{e}}$ \\
\hline 0.0014 & 0.34 & 9.3 & 0.08 & 0.47 & 0.093 & 0.243 & 0.020 & 177.1 & 13.4 & 4.6 \\
0.007 & 1.08 & 7.8 & 0.2 & 0.78 & 0.303 & 0.178 & 0.057 & 212.5 & 14.7 & 5.3 \\
0.014 & 1.59 & 7.2 & 0.28 & 0.88 & 0.447 & 0.163 & 0.083 & 282.5 & 16.9 & 5.4 \\
0.07 & 3.14 & 7.0 & 0.54 & 1.93 & 0.923 & 0.177 & 0.124 & 124.5 & 11.3 & 7.5 \\
0.14 & 5.13 & 7.5 & 0.69 & 3.17 & 1.541 & 0.136 & 0.169 & 122.7 & 11.2 & 9.1 \\
\hline
\end{tabular}

Young's modulus, $E$, fracture strain, $\varepsilon_{b}$, yield stress, $\sigma_{y}$, and fracture stress, $\sigma_{b}$, are obtained from the experimental tensile stress-strain curves; shear modulus of the viscoelastic part, $G_{\mathrm{v}}$, Deborah number, $D_{\mathrm{e}}$, shear modulus of the elastic part, $G_{\mathrm{e}}$, the maximum allowable value of the first strain invariant, $J_{m}$, and theoretical finite extensibility, $\lambda_{m}$, are obtained from theoretical fitting by equation (1) to (4).

The tensile behaviour of the PIC hydrogels can be further analysed by the viscoelastic model proposed by Creton et al., ${ }^{37}$ which was a parallel combination of the Upper Convected Maxwell (UCM) model for the description of viscoelastic flow and the Gent strain hardening model for the entropic elasticity of finite extensibility of polymer chains. This model can capture the physical features of the viscoelastic phase from the dynamic weak ionic bonds and elastic phase from the strong bonds serving as permanent crosslinkers. The model was used to analyse the viscoelasticity of PA hydrogel system. ${ }^{33}$ Herein, we also use this model to analyse the PIC hydrogels. The sum of contributions to the stress, $\sigma_{N}$, from the UCM element and the Gent element can be described as, ${ }^{37}$ 
$\sigma_{N}(\lambda)=\sigma_{N, v}(\lambda)+\sigma_{N, e}(\lambda) \quad \ldots \ldots(1)$

where

$\sigma_{N, v}(\lambda)=\left(\frac{2 G_{v} D_{e}}{1-2 D_{e}}\left(1-\exp \left(-\frac{\left(1-2 D_{e}\right)}{D_{e}}(\lambda-1)\right)+\frac{G_{v} D_{e}}{1+D_{e}}\left(1-\exp \left(-\frac{\left(1+D_{e}\right)}{D_{e}}(\lambda-1)\right)\right) \lambda^{-1} \ldots \ldots\right.\right.$

for the viscoelastic stress and

$$
\sigma_{N, e}(\lambda)=\left(\frac{G_{e}}{1-\frac{\lambda^{2}+2 \lambda^{-1}-3}{J_{m}}}\left(\lambda^{2}-\lambda^{-1}\right)\right) \lambda^{-1}
$$

for the entropic elastic stress, where $G_{v}$ is the initial shear modulus of the viscoelastic part, $D_{e}$ is the Deborah number (the product of the relaxation time of the viscous component and the strain rate), $G_{e}$ is the shear modulus at small strain from the elastic part, and $\lambda$ is the elongation ratio $(\lambda=\varepsilon+1) . J_{m}$ is the maximum allowable value of the first strain invariant, and it is related to the theoretical finite extensibility of the network chains $\lambda_{m}$ as, $J_{m}=\lambda_{m}^{2}+2 \lambda_{m}^{-1}-3$

Eq. 1 is determined by four parameters $G_{e}, \lambda_{m}, G_{v}$, and $D_{e}$. Since the Young's modulus $E=3\left(G_{e}+G_{v}\right)$, we can fit the tensile stress-strain data with the model using three independent parameters and the Young's modulus $E$ estimated from the tensile behaviour. A representative stress-elongation curve for the PIC hydrogel as well as simulated curves of the two individual contributions to the tensile behavior is presented in Figure 6c. A good fit by such a combined model to the tensile data of PIC gels can be obtained. A systematic fitting to the tensile behavior of hydrogels in different tensile rates are performed to understand how the ionic bonds affect the mechanical behavior of the hydrogels, and the related simulated parameters are shown in Table 3. The Deborah 
number, $D_{e}$, in the fitting serves as the adjusting parameter. An importantant parameter, $G_{\mathrm{v}} / G_{\mathrm{e}}$, characterizes the ratio of polymer chains crosslinked by temporary weak bonds and quasi-permanent strong bonds. The value of $G_{\mathrm{v}} / G_{\mathrm{e}}$ also indicates the average number of weak bonds between two neighbouring strong bonds. ${ }^{37}$ Therefore, it is an assessment of the degree of viscoelastic characters and how much energy dissipates occurs during deformation, contributing to toughness. It is clearly shown that the $G_{\mathrm{v}} / G_{\mathrm{e}}$ of all the samples increase with the acceleration of stretch rate, which is consistent with the typical viscoelastic character of PIC hydrogel depending on the stretch rate. In comparison with PA system, the $G_{\mathrm{v}} / G_{\mathrm{e}}$ of the PIC system is high, which is in agreemet with the dynamic rheological results (Figure 4c). ${ }^{33}$ Furthermore, the theoretical finite extensibility of the network chains $\lambda_{\mathrm{m}}$ of the PIC hydrogel at low strain rate $\left(\lambda_{\mathrm{m}} \sim 13\right)$ is much smaller than that of the PA hydrogel $\left(\lambda_{\mathrm{m}} \sim 18\right) .{ }^{33}$ This could be related to the stronger entanglement of the PIC system than that of the PA system, as discussed in the previous section.

\section{Conclusion}

As like the PA hydrogels, the most strong and tough PIC hydrogels are formed near the equal charge ratio and the PIC hydrogels show strong viscoelastic character with wide ionic bonds distribution. For PA hydrogels, the opposite charges, randomly dispersed along the polymer chains, form ionic bonds of a wide strength distribution through interchain and intra-chain complexation. The polyelectrolyte chains are in globule conformation due to intra-chain ionic bonds even at the preparation state, in the presence of large amount of small ions. Because of this, the inter-chain ionic bonds that contribute to the elasticity of the gels could only be formed at extremely high preparation concentration $\left(C_{\mathrm{m}}>1.3 \mathrm{M}\right)$. They show substantial swelling and low modulus at low 
polymer concentration due to poor entanglement between chains $\left(C_{\mathrm{m}}<1.6 \mathrm{M}\right)$. On the other hand, for PIC hydrogels, the homo-charged polymers in the as-prepared state take relatively extended coil conformation because the like-charged segments repel each other and no intra-chain ionic bonds could be formed. This greatly favors the chain entanglement even at relatively low polymer concentration $C_{\mathrm{m}}(>0.2 \mathrm{M})$. By dialysis of the sample in water, the mobile small ions are removed from the gel, and inter-chain complexation between entangled, oppositely charged polymers is formed. Thus, tough PIC gels can be formed at very low concentration $C_{\mathrm{m}}$. Although the primary structure of the PIC hydrogels and PA hydrogels are completely different, the activation energies, which are the energy barriers of the local motion, of the two systems are quite similar. This means that, that the ionic bond strengths at short molecular scale are similar for the two systems. Accordingly, the difference of the mechanical behaviors of the two systems comes from the difference in more large scale structure. SEM observation of the samples morphology supports this argument. The PA shows more uniform structure with small pore size, while the PIC shows a more inhomogeneous, segregated porous structure with large pore size. These differences in large scale structure seem to dominant the bulk mechanical properties of the two systems. On the basis of the different structural character between PA gels and PIC gels, PIC gels possess several advantages such as less $C_{\mathrm{m}}$-dependence, easy reprocessability, and strong self-glued ability. ${ }^{31-32}$

\section{Acknowledgements}

This research was financially supported by Grant-in-Aid for Scientific Research (S) (No. 124225006) and for Postdoctoral Fellowships for Foreign Researchers (No. P12340) 
from the Japan Society for the Promotion of Science (JSPS). This research was also funded by ImPACT Program of Council for Science, Technology and Innovation (Cabinet Office, Government of Japan). 


\section{References}

1. Sun, J. Y.; Zhao, X. H.; Illeperuma, W. R. K.; Chaudhuri, O.; Oh, K. H.; Mooney, D. J.; Vlassak, J. J.; Suo, Z. G., Highly stretchable and tough hydrogels. Nature 2012, 489 (7414), 133-136.

2. Peppas, N. A.; Hilt, J. Z.; Khademhosseini, A.; Langer, R., Hydrogels in biology and medicine: from molecular principles to bionanotechnology. Adv. Mater. 2006, 18 (11), 1345-1360.

3. Nowak, A. P.; Breedveld, V.; Pakstis, L.; Ozbas, B.; Pine, D. J.; Pochan, D.; Deming, T. J., Rapidly recovering hydrogel scaffolds from self-assembling diblock copolypeptide amphiphiles. Nature 2002, 417 (6887), 424-428.

4. $\quad$ Calvert, P., Hydrogels for soft machines. Adv. Mater. 2009, 21 (7), 743-756.

5. Zhao, X., Multi-scale multi-mechanism design of tough hydrogels: building dissipation into stretchy networks. Soft Matter 2014, 10 (5), 672-687.

6. Kim, J.; Hanna, J. A.; Byun, M.; Santangelo, C. D.; Hayward, R. C., Designing responsive buckled surfaces by halftone gel lithography. Science 2012, 335 (6073), 12011205.

7. Henderson, K. J.; Shull, K. R., Effects of Solvent Composition on the Assembly and Relaxation of Triblock Copolymer-Based Polyelectrolyte Gels. Macromolecules 2012, 45 (3), 1631-1635.

8. Li, J.; Illeperuma, W. R.; Suo, Z.; Vlassak, J. J., Hybrid Hydrogels with Extremely High Stiffness and Toughness. Acs Macro Lett. 2014, 3, 520-523.

9. Gong, J. P.; Katsuyama, Y.; Kurokawa, T.; Osada, Y., Double - Network Hydrogels with Extremely High Mechanical Strength. Adv. Mater. 2003, 15 (14), 1155-1158.

10. Wang, J.; Lin, L.; Cheng, Q.; Jiang, L., A Strong Bio - Inspired Layered PNIPAM Clay Nanocomposite Hydrogel. Angewandte Chemie 2012, 124 (19), 4754-4758.

11. Schexnailder, P.; Schmidt, G., Nanocomposite polymer hydrogels. Colloid Polym. Sci. 2009, 287 (1), 1-11.

12. Yang, C. H.; Wang, M. X.; Haider, H.; Yang, J. H.; Sun, J.-Y.; Chen, Y. M.; Zhou, J.; Suo, Z., Strengthening alginate/polyacrylamide hydrogels using various multivalent cations. ACS Appl. Mater. Inter. 2013, 5 (21), 10418-10422.

13. Lin, P.; Ma, S.; Wang, X.; Zhou, F., Molecularly Engineered Dual -Crosslinked Hydrogel with Ultrahigh Mechanical Strength, Toughness, and Good Self - Recovery. Adv. Mater. 2015, 27 (12), 2054-2059.

14. Xia, L.-W.; Xie, R.; Ju, X.-J.; Wang, W.; Chen, Q.; Chu, L.-Y., Nano-structured smart hydrogels with rapid response and high elasticity. Nat.Commun. 2013, 4, 2226.

15. Huang, T.; Xu, H.; Jiao, K.; Zhu, L.; Brown, H. R.; Wang, H., A novel hydrogel with high mechanical strength: a macromolecular microsphere composite hydrogel. Adv. Mater. 2007, 19 (12), 1622-1626.

16. Gong, J. P., Why are double network hydrogels so tough? Soft Matter 2010, 6 (12), 2583-2590. 
17. Cordier, P.; Tournilhac, F.; Soulié-Ziakovic, C.; Leibler, L., Self-healing and thermoreversible rubber from supramolecular assembly. Nature 2008, 451 (7181), 977980.

18. Burnworth, M.; Tang, L.; Kumpfer, J. R.; Duncan, A. J.; Beyer, F. L.; Fiore, G. L.; Rowan, S. J.; Weder, C., Optically healable supramolecular polymers. Nature 2011, 472 (7343), 334-337.

19. Henderson, K. J.; Zhou, T. C.; Otim, K. J.; Shull, K. R., lonically cross-linked triblock copolymer hydrogels with high strength. Macromolecules 2010, 43 (14), 6193-6201.

20. Hunt, J. N.; Feldman, K. E.; Lynd, N. A.; Deek, J.; Campos, L. M.; Spruell, J. M.; Hernandez, B. M.; Kramer, E. J.; Hawker, C. J., Tunable, high modulus hydrogels driven by ionic coacervation. Adv. Mater. 2011, 23 (20), 2327-2331.

21. Chassenieux, C.; Tsitsilianis, C., Recent trends in $\mathrm{pH} /$ thermo-responsive selfassembling hydrogels: from polyions to peptide-based polymeric gelators. Soft Matter 2016, 12, 1344-1359.

22. Dyakonova, M.; Stavrouli, N.; Popescu, M.; Kyriakos, K.; Grillo, I.; Philipp, M.; Jaksch, S.; Tsitsilianis, C.; Papadakis, C., Physical Hydrogels via Charge Driven SelfOrganization of a Triblock Polyampholyte-Rheological and Structural Investigations. Macromolecules 2014, 47 (21), 7561-7572.

23. Lemmers, M.; Sprakel, J.; Voets, I. K.; van der Gucht, J.; Stuart, C.; Martien, A., Multiresponsive Reversible Gels Based on Charge - Driven Assembly. Angewandte Chemie 2010, 122 (4), 720-723.

24. Gao, H.; Yao, H., Shape insensitive optimal adhesion of nanoscale fibrillar structures. P. Natl. Acad. Sci. USA 2004, 101 (21), 7851-7856.

25. Burattini, S.; Colquhoun, H. M.; Fox, J. D.; Friedmann, D.; Greenland, B. W.; Harris, P. J.; Hayes, W.; Mackay, M. E.; Rowan, S. J., A Self-repairing, supramolecular polymer system: healability as a consequence of donor-acceptor $\pi-\pi$ stacking interactions. Chem. Commun. 2009, (44), 6717-6719.

26. Hao, J.; Weiss, R., Viscoelastic and mechanical behavior of hydrophobically modified hydrogels. Macromolecules 2011, 44 (23), 9390-9398.

27. Haque, M. A.; Kurokawa, T.; Kamita, G.; Gong, J. P., Lamellar bilayers as reversible sacrificial bonds to toughen hydrogel: hysteresis, self-recovery, fatigue resistance, and crack blunting. Macromolecules 2011, 44 (22), 8916-8924.

28. Sun, T. L.; Kurokawa, T.; Kuroda, S.; Ihsan, A. B.; Akasaki, T.; Sato, K.; Haque, M. A.; Nakajima, T.; Gong, J. P., Physical hydrogels composed of polyampholytes demonstrate high toughness and viscoelasticity. Nat. Mater. 2013, 12 (10), 932-937.

29. Luo, F.; Sun, T. L.; Nakajima, T.; Kurokawa, T.; Zhao, Y.; Ihsan, A. B.; Guo, H. L.; Li, X. F.; Gong, J. P., Crack Blunting and Advancing Behaviors of Tough and Self-healing Polyampholyte Hydrogel. Macromolecules 2014, 47, 6037-6046.

30. Ihsan, A. B.; Sun, T. L.; Kuroda, S.; Haque, M. A.; Kurokawa, T.; Nakajima, T.; Gong, J. P., A phase diagram of neutral polyampholyte-from solution to tough hydrogel. J. Mater. Chem. B 2013, 1 (36), 4555-4562.

31. Luo, F.; Sun, T. L.; Nakajima, T.; Kurokawa, T.; Zhao, Y.; Sato, K.; Ihsan, A. B.; Li, X.; Guo, H.; Gong, J. P., Oppositely Charged Polyelectrolytes Form Tough, Self - Healing, and Rebuildable Hydrogels. Adv. Mater. 2015, 27 (17), 2722-2727. 
32. Luo, F.; Sun, T. L.; Nakajima, T.; Kurokawa, T.; Ihsan, A. B.; Li, X.; Guo, H.; Gong, J. P., Free Reprocessability of Tough and Self-Healing Hydrogels Based on Polyion Complex. Acs Macro Lett. 2015, 4, 961-964.

33. Sun, T. L.; Luo, F.; Kurokawa, T.; Karobi, S. N.; Nakajima, T.; Gong, J. P., Molecular structure of self-healing polyampholyte hydrogels analyzed from tensile behaviors. Soft Matter 2015, 11, 9355-9366.

34. Klein, J. W., A., J. Polym. Sci., Polym. Chem. Ed. 1981, 19, 707.

35. Dobrynin, A. V.; Colby, R. H.; Rubinstein, M., Scaling theory of polyelectrolyte solutions. Macromolecules 1995, 28 (6), 1859-1871.

36. Mayumi, K.; Marcellan, A.; Ducouret, G.; Creton, C.; Narita, T., Stress-Strain Relationship of Highly Stretchable Dual Cross-Link Gels: Separability of Strain and Time Effect. Acs Macro Lett. 2013, 2 (12), 1065-1068.

37. Deplace, F.; Rabjohns, M. A.; Yamaguchi, T.; Foster, A. B.; Carelli, C.; Lei, C. H.; Ouzineb, K.; Keddie, J. L.; Lovell, P. A.; Creton, C., Deformation and adhesion of a periodic soft-soft nanocomposite designed with structured polymer colloid particles. Soft Matter 2009, 5 (7), 1440-1447. 